\title{
Plasma glutathione reductase activity and prognosis of septic shock
}

\author{
Jae Seong Kim, $\mathrm{MD},{ }^{a}$ Woon Yong Kwon, $\mathrm{MD}, \mathrm{PhD},{ }^{a, *}$ \\ Gil Joon Suh, MD, PhD, ${ }^{a}$ Kyung Su Kim, MD, PhD, ${ }^{a}$ Yoon Sun Jung, MD, ${ }^{b}$ \\ Sung Hee Kim, MSc, ${ }^{a}$ and So Eun Lee, $\mathrm{MD}^{\mathrm{c}}$ \\ ${ }^{a}$ Department of Emergency Medicine, Seoul National University College of Medicine, Seoul, Korea \\ ${ }^{\mathrm{b}}$ Department of Emergency Medicine, National Medical Center, Seoul, Korea \\ ${ }^{\mathrm{c}}$ Department of Emergency Medicine, Incheon Sarang Hospital, Incheon, Korea
}

\section{A R T I C L E I N F O}

Article history:

Received 11 June 2015

Received in revised form

25 July 2015

Accepted 29 July 2015

Available online 5 August 2015

Keywords:

Septic shock

Oxidative stress

Glutathione

Oxidation reduction

Glutathione reductase

Mortality

\begin{abstract}
A B S T R A C T
Background: Our aim was to investigate whether plasma glutathione reductase (GR) activity is well correlated with the erythrocyte-reduced glutathione (GSH)/glutathione disulfide (GSSG) ratio and is associated with the mortality of septic shock.

Materials and methods: This study was conducted on male Sprague-Dawley rats and patients admitted to the intensive care unit with septic shock. To induce endotoxemia in rats, vehicle or lipopolysaccharide (LPS) at dosages of 5 or $10 \mathrm{mg} / \mathrm{kg}$ were injected into a tail vein. Animals were then euthanized $6 \mathrm{~h}$ post-LPS. Based on the 28-d mortality, the enrolled patients were divided into the survivors and nonsurvivors. We obtained blood samples from patients at admission $(0 \mathrm{~h})$ and $24 \mathrm{~h}$ after admission to the intensive care unit.

Results: In endotoxemic rats, the erythrocyte GSH/GSSG ratio, erythrocyte GR activity, and plasma GR activity in the $10 \mathrm{mg} / \mathrm{kg}$ of LPS group were lower than those in the sham and $5 \mathrm{mg} / \mathrm{kg}$ of LPS groups. In patients with septic shock, decrease in plasma GR activity at $24 \mathrm{~h}$ was independently associated with an increase in 28-d mortality (odds ratio, 0.828 ; $95 \%$ confidence interval, 0.690-0.992, $\mathrm{P}=0.041)$. Plasma GR activity was correlated with erythrocyte GR activity (Spearman $\rho=0.549, \mathrm{P}<0.001$ ) and the erythrocyte GSH/GSSG ratio (rho $=0.367, \mathrm{P}=0.009$ ) at $24 \mathrm{~h}$.

Conclusions: Plasma GR activity was well correlated with erythrocyte GR activity and the erythrocyte GSH/GSSG ratio, and a decrease in plasma GR activity was associated with an increase in the mortality of septic shock patients.
\end{abstract}

() 2016 Elsevier Inc. All rights reserved.

\section{Introduction}

\subsection{Background}

Sepsis is defined as a complex clinical syndrome that is caused by a harmful or damaging host response to infection and may progress to the development of multiple-organ dysfunctions and death [1]. Sepsis activates various immune cells and leads to the excessive production of reactive oxygen species (ROS) [2]. The ROS and ROS-derived oxidative stresses appear to be implicated in cellular signal transduction and gene activation, leading to the excessive or inappropriate release of inflammatory mediators and the development of multiorgan failure during sepsis [3-5].

\footnotetext{
* Corresponding author. Department of Emergency Medicine, Seoul National University Hospital, 101 Daehak-ro, Jongno-gu, Seoul 110 744, Korea. Tel.: +82 22072 0326; fax: +82 236728871.

E-mail address: kwy711@hanmail.net (W.Y. Kwon). 0022-4804/\$ - see front matter (c) 2016 Elsevier Inc. All rights reserved.
} 
Oxidative stresses occur when the balance between the production of ROS and their removal by endogenous antioxidant defenses are not maintained [6]. Circulating erythrocytes scavenge extracellular ROS and provide an antioxidant defense for the surrounding tissues [7,8]. Glutathione (GSH) is a major intracellular antioxidant that eliminates ROS [3], and previous studies have reported that oxidative stresses are potentiated by the depletion of reduced GSH during sepsis [9-11]. Intracellular GSH synthesis is mainly regulated by glutamate-cysteine ligase (GCL) activity [12,13]. In erythrocytes, the GSH redox cycle is regulated by glutathione peroxidase (GPx) and glutathione reductase (GR). Briefly, GPx eliminates hydrogen peroxide $\left(\mathrm{H}_{2} \mathrm{O}_{2}\right)$ through the oxidation of GSH to glutathione disulfide (GSSG) $[3,14,15]$. GR then reconverts GSSG to GSH $[3,14,15]$. Therefore, erythrocyte GSH redox (GSH/GSSG) ratio is shown to be a sensitive parameter of antioxidant capacities $[3,11,16]$. To measure the erythrocyte GSH/GSSG ratio, erythrocytes should be separated from blood samples in real time, and many researchers tried to estimate the erythrocyte GSH/ GSSG ratio by whole blood or plasma. Previous clinical studies showed that the blood GSH/GSSG ratio was decreased in patients with septic shock [11]. However, GSH in extracellular areas undergoes spontaneous autoxidation and GSSG formation, and accumulated GSSG in erythrocytes is exported into the plasma [17]. And thus, the blood GSH/GSSG ratio is not fully consistent with the erythrocyte GSH/GSSG ratio, and the erythrocyte GSH/GSSG ratio may not be properly estimated in plasma $[13,16]$.

Among two enzymes which regulate the GSH redox cycle, decreases in plasma GPx activity and the selenium level are known to be associated with poor prognosis of septic shock. Selenium is an essential trace element to synthesize GPx, and previous studies showed that selenium suppressed oxidative stresses by increasing GPx activity [18-20]. However, $\mathrm{GPx}$-induced $\mathrm{H}_{2} \mathrm{O}_{2}$ elimination needs the oxidation of GSH to GSSG. To restore the GSH level and maintain the GSH/GSSG ratio, GSSG should be reduced to GSH. GR catalyzes the reduction of GSSG to GSH mainly in intracellular area and is also secreted into extracellular area and protects cells from external oxidative stresses $[16,21]$. Furthermore, plasma GR activity can be easily measured by spectrophotometry in a clinical setting. However, there have been limited data which investigate whether plasma GR activity is well correlated with the erythrocyte GSH/GSSG ratio and is associated with prognosis of septic shock.

\subsection{Hypotheses and purpose}

Therefore, we hypothesized that erythrocyte GR activity would be well correlated with the erythrocyte GSH/GSSG ratio, plasma GR activity would be well correlated with the erythrocyte GR activity, and thus plasma GR activity would be well correlated with the erythrocyte GSH/GSSG ratio. We also hypothesized that a decrease in plasma GR activity would be associated with an increase in the mortality of patients with septic shock. To verify our hypothesis, we performed this study.

\section{Materials and methods}

\subsection{Ethics statement}

All the conducted animal experiments were approved by the Institutional Animal Care and Use Committee of Seoul National University Hospital (IACUC Number: 13-0393-S1A0) in accordance with the NIH Guide for the Care and Use of Laboratory Animals.

We obtained clinical data and blood samples from the consecutive patients with septic shock who enrolled in a prospective cohort study, the repository for sepsis and postresuscitation samples (NCT01670383), from September 2012-April 2013. This study was performed in compliance with the Declaration of Helsinki (Seoul, Korea, 2008) and was approved by the Institutional Review Board of Seoul National University College of Medicine/Seoul National University Hospital (institutional review board number: 1012-140-347 and 1312-028-539). All the patients or their representatives provided written informed consent.

\subsection{Animals and drugs}

The experiments were performed on male Sprague-Dawley rats (body weight, 300-350 g) purchased from Orient Bio Inc (Seongnam, Korea). Estrogen is known to influence pathophysiology and prognosis of sepsis [22,23]. Therefore, we did not use female gender rats in animal experimental study to avoid confounding effects by different phases of estrous cycle. However, in the clinical study, we collected data from both the male and female septic shock patients. The animals were provided with a laboratory chow (Lab Diet, Seongnam, Korea) and water ad libitum and were housed in a specific pathogenfree room at constant temperature $\left(20^{\circ} \mathrm{C}-22^{\circ} \mathrm{C}\right)$ with 10 and $14 \mathrm{~h}$ of light and dark exposure, respectively. The animals underwent an acclimatization period of $14 \mathrm{~d}$ before being used in the experiments.

Lipopolysaccharide (LPS, from Escherichia coli, O26:B6) was purchased from Sigma-Aldrich Chemical (St Louis, MO).

\subsection{Animal experimental procedures}

To induce endotoxemia in rats, vehicle or LPS at dosages of 5 or $10 \mathrm{mg} / \mathrm{kg}$ were injected into a tail vein. The animals were then returned to their cages and were allowed food and water ad libitum. The subjects were divided into three groups as follows: (1) the rats in the sham group were administered a vehicle intravenously; (2) the rats in the low dose of LPS (LDLPS) group were administered $5 \mathrm{mg} / \mathrm{kg}$ of LPS intravenously; and (3) the rats in the high dose of LPS (HD-LPS) group were administered $10 \mathrm{mg} / \mathrm{kg}$ of LPS intravenously.

For the survival analysis, the animals were allocated to the sham group $(n=6)$, the LD-LPS group, or the HD-LPS group ( $n=11$ per group) and were closely observed for the occurrence of mortality during a period of $72 \mathrm{~h}$. To evaluate the effects of low and high doses of LPS, a separate set of animals was allocated to the study groups based on the survival study findings: the sham group $(n=6)$, the LD-LPS group $(n=8)$, and 
the HD-LPS group $(n=10)$. We sacrificed six animals in each group and obtained blood samples by cardiac puncture at $6 \mathrm{~h}$ post-LPS administration.

\subsection{Patients}

When patients met the diagnostic criteria of septic shock in the Emergency Department, they were immediately admitted to the intensive care unit (ICU). The exclusion criteria were pregnancy, age $<18 \mathrm{y}$, death within $24 \mathrm{~h}$ of the diagnosis of septic shock, presence of advance directives to withhold or withdraw life-sustaining treatment, or no informed consent. Septic shock was defined as sepsis with organ dysfunction and sepsis-induced hypotension (systolic blood pressure $<90 \mathrm{~mm} \mathrm{Hg}$ or mean arterial pressure [MAP] $<70 \mathrm{~mm} \mathrm{Hg}$ ) despite adequate fluid resuscitation $(20-30 \mathrm{~mL} / \mathrm{kg}$ of crystalloid) [24]. Organ dysfunction was defined as follows: altered mental status, arterial hypoxemia $\left(\mathrm{PaO}_{2} / \mathrm{FiO}_{2}<300\right)$, acute oliguria (urine output $<0.5 \mathrm{~mL} / \mathrm{kg} / \mathrm{h}$ for at least $2 \mathrm{~h}$ ), creatinine increase $(>0.5 \mathrm{mg} / \mathrm{dL})$, coagulation abnormalities (international normalized ratio of prothrombin time [PT] $>1.5$ or activated partial thromboplastin time [aPTT] $>60 \mathrm{~s}$ ), ileus (absent bowel sounds), thrombocytopenia (platelet count $<100,000 / \mu \mathrm{L}$ ), hyperbilirubinemia (plasma total bilirubin $>4 \mathrm{mg} / \mathrm{dL}$ ), hyperlactatemia (>1 mmol/L), or decreased capillary refill or mottling [24].

When patients were diagnosed as septic shock, they received $20-30 \mathrm{~mL} / \mathrm{kg}$ of crystalloid immediately and were managed based on the "Surviving Sepsis Campaign: international guidelines for management of severe sepsis and septic shock: 2008" [25]. Briefly, the management protocol included fluid resuscitation to achieve a central venous pressure (CVP) of 8-12 mm $\mathrm{Hg}$, vasoactive agents to achieve a MAP of 65-90 $\mathrm{mm} \mathrm{Hg}$, red blood cell transfusion or dobutamine infusion to achieve a central venous oxygen saturation $\left(\mathrm{ScvO}_{2}\right)$ of $70 \%$ or greater, blood and other sites cultures with broad-spectrum antibiotics, hydrocortisone for refractory shock, and intravenous insulin for glucose control with a target $<150 \mathrm{mg} / \mathrm{dL}$. The primary outcome was defined as death occurring within $28 \mathrm{~d}$ of admission. Based on the 28-d mortality, the enrolled patients were divided into two groups: the survivors and the nonsurvivors.

At the time of admission to the ICU, we collected demographic data. We calculated the Acute Physiology and Chronic Health Evaluation II (APACHE II) score and the sequential organ failure assessment score from the data collected during the first 24-h admission period [26,27]. We obtained blood samples through an arterial catheter at admission $(0 \mathrm{~h})$ and $24 \mathrm{~h}$ after admission to the ICU.

\subsection{Blood sample preparation}

Blood samples were centrifuged at $2000 \mathrm{~g}$ for $15 \mathrm{~min}$ at $4^{\circ} \mathrm{C}$, and the separated plasma were stored at $-80^{\circ} \mathrm{C}$ until further analyses. After the plasma separation, the white buffy layers were removed, and the erythrocytes were lysed in ice-cold high performance liquid chromatography-grade water. The erythrocyte lysates were centrifuged at $10,000 \mathrm{~g}$ for $15 \mathrm{~min}$ at $4^{\circ} \mathrm{C}$, and the supernatants were collected and stored at $-80^{\circ} \mathrm{C}$ until further analyses.

\subsection{Measurements of antioxidants and oxidative stresses}

The plasma total glutathione (T-GSH) level and the erythrocyte GSH and GSSG levels were measured using a glutathione assay kit (Cayman Chem, Ann Arbor, MI). Plasma and erythrocyte GR activities were measured using a GSH reductase assay kit (Cayman Chem), and plasma and erythrocyte GPx activities were measured using a glutathione peroxidase assay kit (Cayman Chem) according to the manufacturer's instructions. The erythrocyte glutamate-cysteine ligase, catalytic subunit (GCLC) level was measured using a GCLC ELISA kit (MyBioSource, Inc, San Diego, CA) [28].

As a parameter to estimate systemic oxidative stresses, we measured the plasma malondialdehyde (MDA) level using a Lipid Peroxidation (MDA) Assay Kit (Colorimetric/Fluorometric; Abcam, Cambridge, MA) [29]. To evaluate the ability of the plasma MDA level to estimate oxidative stresses, the erythrocyte $\mathrm{H}_{2} \mathrm{O}_{2}$ level was measured in rats using a hydrogen peroxide colorimetric detection kit (Enzo Life Science, Farmingdale, NY), and then its correlation with the plasma MDA level was analyzed.

In addition, the plasma selenium level was measured by electrothermal atomic absorption spectrometry using a spectrometer equipped with an Atomic Absorption Spectrophotometer (AAS 800; PerkinElmer Inc, San Jose, CA) [30].

\subsection{Western blot}

To determine the expressions of plasma and erythrocyte GR and GPx in rats, we performed Western blotting. The following primary antibodies were used: rabbit anti-rat-GSH reductase (diluted 1:1000; Abcam) and anti-rat-glutathione peroxidase (diluted 1:1000; Abcam). The secondary antibodies used were anti-rabbit immunoglobulin $\mathrm{G}$ (Enzo Life Science) coupled with peroxidase and diluted 1:2000 in Tris-buffered saline-Tween. To ensure equivalent protein loading from plasma (20 $\mu \mathrm{g}$ per lane) and erythrocyte lysate (10 $\mu \mathrm{g}$ per lane), the blots were normalized against $\beta$-actin. For $\beta$-actin measurement, mouse monoclonal anti- $\beta$-actin (Cell Signaling, Beverly, CA) was used.

\subsection{Statistical analysis}

The rat survival data were compared using the Kaplan-Meier survival analysis and the log-rank test. The other experimental data were analyzed by the Kruskal-Wallis test with the Mann-Whitney $U$ post hoc test and Bonferroni correction. Moreover, correlations between the parameters were analyzed using Spearman correlation analysis.

The clinical data were compared using the Mann-Whitney $U$ test, the chi-square test, or Fisher exact test as appropriate. The time-related effects of the hemodynamic, plasma, and erythrocyte parameters on the 28-d mortality of septic shock patients were analyzed using the logistic regression and stepwise logistic regression analyses (with an entry level of 0.05 and a stay level of 0.05 ), respectively. The linearity assumption of continuous variables was investigated using restricted cubic splines [31]. To investigate which parameters are independently associated with an increase in the 28- $d$ mortality of patients with septic shock, the multivariable 
analysis using the stepwise logistic regression analysis (with an entry level of 0.05 and a stay level of 0.05 ) was then applied to the variables which showed statistical differences between the 28- $d$ survivors and the nonsurvivors.

$P$ values of $<0.05$ were considered to be statistically significant, and the significance levels quoted are two sided. The statistical analyses were conducted using SPSS version 21.0 for Windows (SPSS, Chicago, IL) and SAS version 9.2 (SAS Institute, Cary, NC) and were reviewed by the Medical Research Collaborating Center of Seoul National University Hospital.

\section{Results}

\subsection{Survival of endotoxemic rats}

No mortality occurred in the sham group. In the LD-LPS and HD-LPS groups, 8 of 11 (72.7\%) and 2 of 11 (18.2\%) rats remained alive for $72 \mathrm{~h}$, respectively. The survival in the
LD-LPS group was significantly higher than that in the HD-LPS group ( $P=0.007$; Fig. $1 \mathrm{~A})$.

\subsection{Plasma and erythrocyte parameters in endotoxemic rats}

There were no differences in the hemoglobin level $(P=0.907)$ between the groups (Supplemental Table 1). The plasma T-GSH level in the HD-LPS group was lower than that in the sham group $(P=0.006$; Fig. $1 B)$. The erythrocyte GSH/GSSG ratio in the HD-LPS group was lower than that in the sham $(P=0.006)$ and LD-LPS groups $(P=0.006$; Fig. $1 B)$. The erythrocyte GSSG level in the HD-LPS group was higher than that in the sham $(P=0.006)$ and LD-LPS groups $(P=0.027$; Supplemental Table 1).

The plasma GR activity in the HD-LPS group was lower than that in the sham $(P=0.006)$ and LD-LPS groups $(P=0.027)$, and the plasma GR activity in the LD-LPS group was also lower than that in the sham group $(P=0.006$; Fig. $1 C)$. The plasma
A

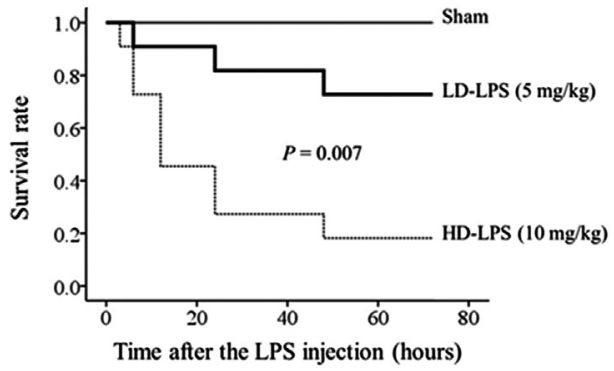

B

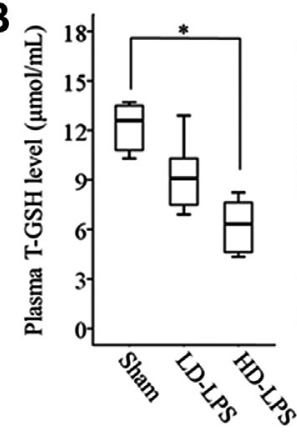

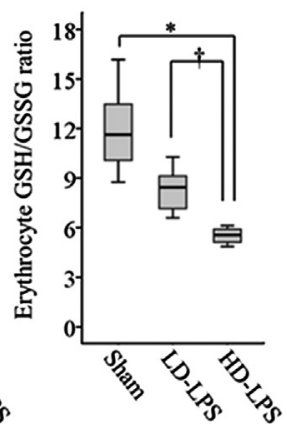
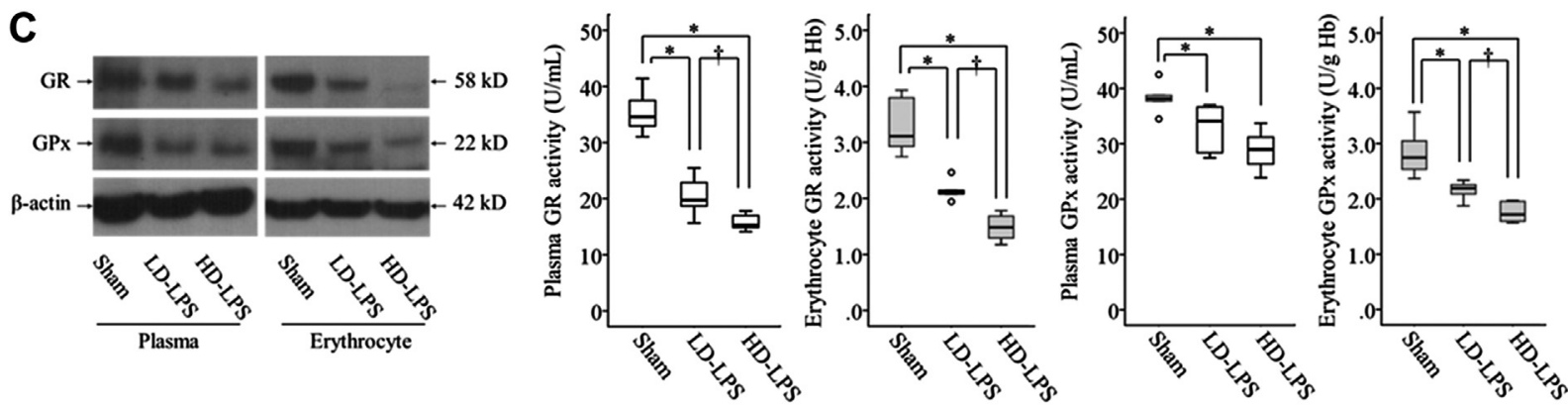

D

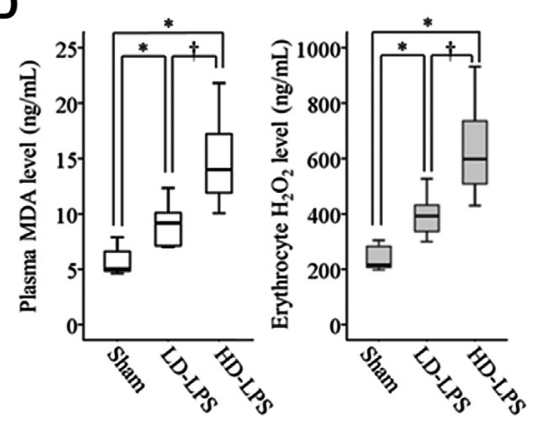

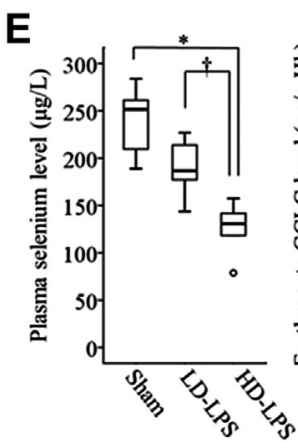

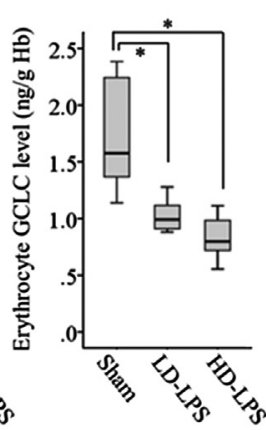

Fig. 1 - Animal experimental data. (A) The survival in the LD-LPS (5 mg/kg) group was significantly higher than that in the HD-LPS (10 mg/kg) group. The LPS injection decreased (B) the plasma T-GSH level; the erythrocyte reduced GSH/GSSG ratio, (C) and the plasma and erythrocyte expressions and activities of GR and GPx. Blots are representative of three rats per group. The LPS injection increased (D) the plasma MDA and erythrocyte $\mathrm{H}_{2} \mathrm{O}_{2}$ levels but decreased (E) the plasma selenium and erythrocyte GCLC levels. Data are presented as median (quartile, range). ${ }^{*}$ Adjusted $P<0.05$ versus the sham group.

${ }^{\dagger}$ Adjusted $P<0.05$ versus the LD-LPS group. 
Table 1 - Demographic characteristics of enrolled patients.

\begin{tabular}{|c|c|c|c|}
\hline \multirow[t]{2}{*}{ Characteristics } & \multicolumn{2}{|c|}{ Median (range) } & \multirow[t]{2}{*}{$P$} \\
\hline & Survivors $(n=34)$ & Nonsurvivors $(n=16)$ & \\
\hline Age, y & $67.5(29.0-87.0)$ & $73.5(51.0-86.0)$ & 0.061 \\
\hline Male gender, $n(\%)$ & $21(61.8)$ & $11(68.8)$ & 0.631 \\
\hline \multicolumn{4}{|l|}{ Comorbidities on admission, $n(\%)$} \\
\hline Diabetes mellitus & $10(29.4)$ & $3(18.8)$ & 0.508 \\
\hline Chronic liver diseases & $5(14.7)$ & $4(25.0)$ & 0.442 \\
\hline Cardiovascular diseases & $5(14.7)$ & $1(6.3)$ & 0.650 \\
\hline Chronic lung diseases & $2(5.9)$ & $4(25.0)$ & 0.074 \\
\hline Chronic renal failure & $4(11.8)$ & $2(12.5)$ & 1.000 \\
\hline Cancer & $8(23.5)$ & $6(37.5)$ & 0.305 \\
\hline Immunosuppression & $4(11.8)$ & $4(25.0)$ & 0.249 \\
\hline \multicolumn{4}{|l|}{ Site of infection, $n(\%)$} \\
\hline Respiratory & $9(26.5)$ & $8(50.0)$ & 0.101 \\
\hline Gastrointestinal & $4(11.8)$ & $3(18.8)$ & 0.666 \\
\hline Hepatobiliary & $9(26.5)$ & $3(18.8)$ & 0.728 \\
\hline Urogenital & $6(17.6)$ & $2(12.5)$ & 1.000 \\
\hline Bone and soft tissue & $5(14.7)$ & $0(0.0)$ & 0.163 \\
\hline Others ${ }^{\dagger}$ & $1(2.9)$ & $0(0.0)$ & 1.000 \\
\hline Bacteremia, $n(\%)$ & $16(47.1)$ & $9(56.3)$ & 0.544 \\
\hline \multicolumn{4}{|l|}{ Pathogen of infection, $n(\%)$} \\
\hline Gram positive & $8(23.5)$ & $3(18.8)$ & 1.000 \\
\hline Gram negative & $20(58.8)$ & $8(50.0)$ & 0.558 \\
\hline Fungal & $1(2.9)$ & $2(12.5)$ & 0.237 \\
\hline Unknown & $5(14.7)$ & $3(18.8)$ & 0.699 \\
\hline APACHE II score for $24 \mathrm{~h}$ after admission & $21.0(5.0-45.0)$ & $32.0(22.0-48.0)$ & $<0.001$ \\
\hline SOFA score for $24 \mathrm{~h}$ after admission & $10.0(3.0-19.0)$ & $14.5(6.0-20.0)$ & $<0.001$ \\
\hline Respiration & $2.0(0.0-2.0)$ & $3.0(0.0-3.0)$ & 0.001 \\
\hline Neurological & $1.0(0.0-4.0)$ & $4.0(0.0-4.0)$ & $<0.001$ \\
\hline Cardiovascular & $4.0(0.0-4.0)$ & $4.0(4.0-4.0)$ & 0.053 \\
\hline Liver & $0.5(0.0-4.0)$ & $2.0(0.0-4.0)$ & 0.044 \\
\hline Coagulation & $1.0(0.0-4.0)$ & $2.0(0.0-3.0)$ & 0.402 \\
\hline Renal & $1.0(0.0-4.0)$ & $2.0(0.0-3.0)$ & 0.211 \\
\hline Interval from diagnosis to first antibiotics, $h$ & $2.0(1.0-5.0)$ & $1.25(1.0-5.0)$ & 0.715 \\
\hline \multicolumn{4}{|l|}{ Managements, $n(\%)$} \\
\hline Mechanical ventilator care & $18(52.9)$ & $10(62.5)$ & 0.525 \\
\hline Renal replacement therapy & 7 (20.6) & 7 (43.8) & 0.089 \\
\hline Hydrocortisone use & $13(38.2)$ & $8(50.0)$ & 0.432 \\
\hline Surgical intervention & $2(5.9)$ & $0(0.0)$ & 0.458 \\
\hline Radiologic intervention & $10(29.4)$ & $4(25.0)$ & 0.513 \\
\hline
\end{tabular}

SOFA score = sequential organ failure assessment score.

"Defined as neutropenia (neutrophil count $<500 / \mu \mathrm{L}$ ), steroid therapy for at least $10 \mathrm{~d}$, chemotherapy during the last $30 \mathrm{~d}$, or therapy with immunosuppressive drugs.

${ }^{\dagger}$ Includes central nervous system and indwelling catheter.

GPx activities in the HD-LPS $(P=0.006)$ and LD-LPS groups $(P=0.045)$ were lower than those in the sham group (Fig. 1C). The erythrocyte GR and GPx activities in the HD-LPS group were lower than those in the sham $(P=0.006$ and 0.006 , respectively) and LD-LPS groups $(P=0.006$ and $P=0.027$; Fig. 1C). The erythrocyte GR and GPx activities in the LD-LPS group were also lower than those in the sham group $(P=0.006$ and $P=0.006$; Fig. $1 C)$.

The plasma MDA and the erythrocyte $\mathrm{H}_{2} \mathrm{O}_{2}$ levels in the HD-LPS group were higher than those in the sham $(P=0.006$ and $P=0.006)$ and LD-LPS groups $(P=0.045$ and $P=0.045$; Fig. 1D). The plasma MDA and the erythrocyte $\mathrm{H}_{2} \mathrm{O}_{2}$ levels in the LD-LPS group were also lower than those in the sham group $(P=0.027$ and $P=0.012$; Fig. $1 D)$.

The plasma selenium and the erythrocyte GCLC levels in the HD-LPS group were lower than those in the sham group
$(P=0.006$ and $P=0.006$; Fig. $1 E)$. The plasma selenium level in the HD-LPS group was also lower than that in the LD-LPS group $(P=0.012)$, and the erythrocyte GCLC level in the LDLPS group was lower than that in the sham group $(P=0.012$; Fig. 1E).

\subsection{Demographic characteristics of enrolled patients}

During the study period, 61 patients with septic shock were admitted to the ICU. Of these patients, 11 were excluded, and a total of 50 were enrolled in this study (Supplemental Fig. 1). Among them, 34 patients (68.0\%) were 28 -d survivors and 16 (32.0\%) were nonsurvivors.

The APACHE II score $(P<0.001)$ and the sequential organ failure assessment score $(P<0.001)$ for $24 \mathrm{~h}$ after admission in the survivors were lower than those in the nonsurvivors 
Table 2 - Effects of hemodynamic and plasma parameters on mortality of septic shock patients.

\begin{tabular}{|c|c|c|c|c|c|c|c|c|c|c|c|}
\hline \multirow[t]{2}{*}{ Parameter } & \multirow[t]{2}{*}{ Hour } & \multicolumn{2}{|c|}{$\begin{array}{l}\text { Survivors } \\
(n=34)\end{array}$} & \multicolumn{2}{|c|}{$\begin{array}{l}\text { Nonsurvivors } \\
\quad(n=16)\end{array}$} & \multicolumn{3}{|c|}{$\begin{array}{l}\text { Univariate } \\
\text { analysis }\end{array}$} & \multicolumn{3}{|c|}{$\begin{array}{l}\text { Stepwise } \\
\text { regression }\end{array}$} \\
\hline & & Median & Range & Median & Range & OR & $95 \% \mathrm{CI}$ & $P$ & OR & $95 \% \mathrm{CI}$ & $P$ \\
\hline \multicolumn{12}{|l|}{ Hemodynamic parameters } \\
\hline \multirow[t]{2}{*}{ MAP (mm Hg) } & 0 & 62.5 & $37.0-104.0$ & 68.0 & $34.0-120.0$ & 1.016 & $0.982-1.051$ & 0.370 & & & \\
\hline & 6 & 86.0 & $58.00-112.0$ & 77.0 & $60.0-106.0$ & 0.962 & $0.917-1.008$ & 0.103 & & & \\
\hline \multirow[t]{2}{*}{ CVP (mm Hg) } & 0 & 8.0 & $3.0-26.0$ & 10.0 & $3.0-23.0$ & 1.053 & $0.953-1.163$ & 0.311 & & & \\
\hline & 6 & 8.0 & $2.0-25.0$ & 10.5 & $3.0-26.0$ & 1.086 & $0.978-1.206$ & 0.125 & & & \\
\hline \multirow[t]{2}{*}{$\mathrm{ScvO}_{2}(\%)$} & 0 & 71.5 & $46.4-91.0$ & 71.7 & $52.9-91.0$ & 1.010 & $0.953-1.071$ & 0.734 & & & \\
\hline & 6 & 75.9 & $50.0-98.0$ & 71.5 & $53.7-86.0$ & 0.951 & $0.880-1.028$ & 0.207 & & & \\
\hline \multirow[t]{2}{*}{ Lactate level (mmol/L) } & 0 & 2.7 & $0.8-12.2$ & 5.2 & $1.0-15.5$ & 1.256 & $1.063-1.484$ & 0.007 & & & \\
\hline & 6 & 2.7 & $0.6-14.1$ & 4.8 & $1.4-15.5$ & 1.292 & $1.083-1.542$ & 0.004 & 1.292 & $1.083-1.542$ & 0.004 \\
\hline \multicolumn{12}{|l|}{ Plasma parameters } \\
\hline \multirow{2}{*}{ T-GSH level ${ }^{\ddagger}(\mu \mathrm{mol} / \mathrm{L})$} & 0 & 0.47 & $0.11-1.54$ & 0.45 & $0.13-0.75$ & 0.915 & $0.743-1.126$ & 0.402 & & & \\
\hline & 24 & 0.99 & $0.66-2.00$ & 0.83 & $0.10-1.05$ & 0.624 & $0.432-0.901$ & 0.012 & 0.624 & $0.432-0.901$ & 0.012 \\
\hline \multirow[t]{2}{*}{ GR activity (U/mL) } & 0 & 9.68 & $3.06-34.64$ & 7.58 & $3.06-16.11$ & 0.874 & $0.754-1.014$ & 0.076 & & & \\
\hline & 24 & 18.43 & $3.13-98.82$ & 8.76 & $3.06-21.21$ & 0.850 & $0.758-0.952$ & 0.005 & 0.850 & $0.758-0.952$ & 0.005 \\
\hline \multirow[t]{2}{*}{ GPx activity (U/mL) } & 0 & 41.82 & $28.42-72.23$ & 39.89 & $29.24-64.93$ & 0.985 & $0.924-1.049$ & 0.629 & & & \\
\hline & 24 & 66.65 & $38.66-106.77$ & 53.50 & $33.01-85.66$ & 0.957 & $0.918-0.997$ & 0.037 & 0.957 & $0.918-0.997$ & 0.037 \\
\hline \multirow[t]{2}{*}{ Sel level ( $\mu \mathrm{g} / \mathrm{L})$} & 0 & 59.50 & $38.00-91.00$ & 43.50 & $28.00-94.00$ & 0.969 & $0.932-1.008$ & 0.114 & & & \\
\hline & 24 & 53.00 & $36.00-88.00$ & 44.50 & $21.00-71.00$ & 0.952 & $0.909-0.996$ & 0.033 & 0.952 & $0.909-0.996$ & 0.033 \\
\hline \multirow[t]{2}{*}{ MDA level (ng/mL) } & 0 & 0.32 & $0.00-4.64$ & 0.56 & $0.01-6.71$ & 1.129 & $0.776-1.641$ & 0.526 & & & \\
\hline & 24 & 0.09 & $0.00-3.22$ & 1.18 & $0.26-7.25$ & 2.842 & $1.345-6.006$ & 0.006 & 2.842 & $1.345-6.006$ & 0.006 \\
\hline
\end{tabular}

Sel $=$ selenium; $0 \mathrm{~h}=$ at admission; $6 \mathrm{~h}=6 \mathrm{~h}$ after admission; $24 \mathrm{~h}=24 \mathrm{~h}$ after admission.

*Analyzed using the logistic regression analysis.

Analyzed using the stepwise logistic regression analysis (entry level 0.05 and stay level 0.05 ).

For univariate and stepwise regression analyses, multiplied by 10 .

(Table 1). In terms of the other demographic characteristics, no significant differences were observed between the two groups (Table 1). The first antibiotics were administered at $1.75 \mathrm{~h}$ after diagnosis.

\subsection{Effects of hemodynamic and plasma parameters on mortality of septic shock patients}

In hemodynamic parameters, an increase in the plasma lactate level at $6 \mathrm{~h}$ (odds ratio [OR], 1.292; 95\% confidence interval [CI], 1.083-1.542; $P=0.004$ ) was associated with an increase in the 28-d mortality of patients with septic shock (Table 2). However, MAP, CVP, and $\mathrm{ScvO}_{2}$ at 0 and $6 \mathrm{~h}$ were not associated with mortality (Table 2). The median values (range) of MAP, CVP, and $\mathrm{ScvO}_{2}$ at $6 \mathrm{~h}$ after diagnosis were $81.0 \mathrm{~mm} \mathrm{Hg}$ (58.0-112.0), $9.5 \mathrm{~mm} \mathrm{Hg}(2.0-26.0)$, and $75.2 \%$ (50.0-98.0), respectively.

In plasma parameters, decreases in the plasma T-GSH level at $24 \mathrm{~h}(\mathrm{OR}, 0.624 ; 95 \% \mathrm{CI}, 0.432-0.901 ; \mathrm{P}=0.012)$, GR activities at $24 \mathrm{~h}(\mathrm{OR}, 0.850 ; 95 \% \mathrm{CI}, 0.758-0.952 ; \mathrm{P}=0.005)$, GPx activity at $24 \mathrm{~h}$ (OR, 0.957; 95\% CI, 0.918-0.997; $\mathrm{P}=0.037$ ), and the selenium level at $24 \mathrm{~h}(\mathrm{OR}, 0.952 ; 95 \% \mathrm{CI}, 0.909-0.996 ; \mathrm{P}=0.033)$ were associated with an increase in the 28-d mortality of patients with septic shock (Table 2). Moreover, an increase in the MDA level at $24 \mathrm{~h}(\mathrm{OR}, 2.842 ; 95 \% \mathrm{CI}, 1.345-6.006 ; \mathrm{P}=0.006)$ was associated with an increase in mortality (Table 2).

Table 3 - Multivariable analysis of hemodynamic and plasma parameters for mortality of septic shock patients.

\begin{tabular}{|c|c|c|c|c|c|c|}
\hline \multirow[t]{2}{*}{ Parameter } & \multicolumn{3}{|c|}{ Univariate analysis ${ }^{*}$} & \multicolumn{3}{|c|}{ Multivariable analysis ${ }^{\dagger}$} \\
\hline & OR & $95 \%$ CI & $P$ & OR & $95 \%$ CI & $P$ \\
\hline Age & 1.059 & $(0.994-1.128)$ & 0.077 & & & \\
\hline APACHE II score for $24 \mathrm{~h}$ after admission & 1.205 & $(1.080-1.345)$ & 0.001 & 1.401 & $(1.078-1.802)$ & 0.012 \\
\hline SOFA score for $24 \mathrm{~h}$ after admission & 1.430 & $(1.143-1.789)$ & 0.002 & & & \\
\hline Plasma lactate level at $6 \mathrm{~h}$ after admission & 1.292 & $(1.083-1.542)$ & 0.004 & & & \\
\hline Plasma T-GSH level at $24 \mathrm{~h}$ after admission & 0.624 & $(0.432-0.901)$ & 0.012 & & & \\
\hline Plasma GR activity at $24 \mathrm{~h}$ after admission & 0.850 & $(0.758-0.952)$ & 0.005 & 0.828 & $(0.690-0.992)$ & 0.041 \\
\hline Plasma GPx activity at $24 \mathrm{~h}$ after admission & 0.957 & $(0.918-0.997)$ & 0.037 & & & \\
\hline Plasma selenium level at $24 \mathrm{~h}$ after admission & 0.952 & $(0.909-0.996)$ & 0.033 & 0.842 & $(0.719-0.987)$ & 0.034 \\
\hline Plasma MDA level at $24 \mathrm{~h}$ after admission & 2.842 & $(1.345-6.006)$ & 0.006 & & & \\
\hline
\end{tabular}

SOFA score = sequential organ failure assessment score.

*Analyzed using the logistic regression analysis.

${ }^{\dagger}$ Analyzed using the stepwise logistic regression analysis (entry level 0.05 and stay level 0.05 ).

${ }^{\ddagger}$ For univariate and multivariable analyses, multiplied by 10. 
Table 4 - Effects of erythrocyte parameters on mortality of septic shock patients.

\begin{tabular}{|c|c|c|c|c|c|c|c|c|c|c|c|}
\hline \multirow[t]{2}{*}{ Parameter } & \multirow[t]{2}{*}{ Hour } & \multicolumn{2}{|c|}{ Survivors $(n=34)$} & \multicolumn{2}{|c|}{ Nonsurvivors $(n=16)$} & \multicolumn{3}{|c|}{ Univariate analysis } & \multicolumn{3}{|c|}{ Stepwise regression ${ }^{\dagger}$} \\
\hline & & Median & Range & Median & Range & OR & $95 \%$ CI & $P$ & OR & $95 \%$ CI & $P$ \\
\hline \multirow[t]{2}{*}{ GSH/GSSG ratio } & 0 & 11.22 & $2.30-27.90$ & 10.47 & $3.93-17.23$ & 1.017 & $0.901-1.149$ & 0.781 & & & \\
\hline & 24 & 11.47 & $4.91-29.71$ & 7.79 & $4.52-17.31$ & 0.717 & $0.568-0.905$ & 0.005 & 0.717 & $0.568-0.905$ & 0.005 \\
\hline \multirow[t]{2}{*}{ GR activity (U/g Hb) } & 0 & 1.92 & $0.81-5.06$ & 1.70 & $0.66-3.76$ & 0.645 & $0.359-1.159$ & 0.143 & & & \\
\hline & 24 & 3.39 & $1.35-7.94$ & 2.94 & $1.11-3.38$ & 0.314 & $0.138-0.714$ & 0.006 & 0.314 & $0.138-0.714$ & 0.006 \\
\hline \multirow[t]{2}{*}{ GPx activity (U/g Hb) } & 0 & 5.89 & $2.97-10.36$ & 4.83 & $2.98-7.54$ & 0.695 & $0.474-1.021$ & 0.064 & & & \\
\hline & 24 & 6.55 & $4.49-10.47$ & 5.74 & $2.87-9.98$ & 0.637 & $0.406-1.000$ & 0.050 & 0.637 & $0.406-1.000$ & 0.050 \\
\hline \multirow[t]{2}{*}{ GCLC level (ng/g Hb) } & 0 & 1.96 & $0.64-4.86$ & 1.67 & $0.73-3.80$ & 0.514 & $0.245-1.080$ & 0.079 & & & \\
\hline & 24 & 21.82 & $5.88-32.98$ & 12.13 & $5.91-24.25$ & 0.902 & $0.830-0.979$ & 0.014 & 0.902 & $0.830-0.979$ & 0.014 \\
\hline
\end{tabular}

3.5. Multivariable analysis of hemodynamic and plasma parameters for mortality of septic shock patients

In the multivariable analysis, an increase in the APACHE II score (OR, 1.401; 95\% CI, 1.078-1.802; $P=0.012$ ) and decreases in plasma GR activity at $24 \mathrm{~h}$ (OR, 0.828; 95\% CI, 0.690-0.992; $\mathrm{P}=0.041)$ and the plasma selenium level at $24 \mathrm{~h}(\mathrm{OR}, 0.842 ; 95 \%$ CI, $0.719-0.987 ; P=0.034$ ) were independently associated with an increase in the 28- $d$ mortality of patients with septic shock (Table 3).

\subsection{Effects of erythrocyte parameters on mortality of septic shock patients}

In erythrocyte parameters, decreases in the erythrocyte GSH/ GSSG level at $24 \mathrm{~h}$ (OR, 0.717; 95\% CI, 0.568-0.905; P = 0.005), GR activity at $24 \mathrm{~h}$ (OR, 0.314; 95\% CI, 0.138-0.714; $P=0.006)$, GPx activity at $24 \mathrm{~h}(\mathrm{OR}, 0.637 ; 95 \% \mathrm{CI}, 0.406-1.000 ; \mathrm{P}=0.050)$, and the GCLC level at $24 \mathrm{~h}(\mathrm{OR}, 0.902 ; 95 \% \mathrm{CI}, 0.830-0.979 ; \mathrm{P}=0.014)$ were associated with an increase in the 28 - $d$ mortality of patients with septic shock (Table 4). There was no difference in the serially measured hemoglobin levels between the survivors and the nonsurvivors (Supplemental Table 2). A decrease in the erythrocyte GSH/GSSG level at $24 \mathrm{~h}$ was contributed by a decrease in the GSH level (OR, 0.828; 95\% CI, 0.713-0.962; $P=0.014)$ and an increase in the GSSG level (OR, 2.261; 95\% CI, 1.250-4.093; $P=0.007$; Supplemental Table 2).

\subsection{Correlations between the 24-h plasma and} erythrocyte parameters of septic shock patients

At $24 \mathrm{~h}$, the effects of plasma and erythrocyte parameters on the 28-d mortality of patients with septic shock were significant, and, thus, we evaluated the correlations between the 24$\mathrm{h}$ parameters. Erythrocyte GR activity was correlated with the erythrocyte GSH/GSSG ratio (Spearman $\rho=0.649, \mathrm{P}<0.001$; Fig. 2A). Plasma GR activity was correlated with erythrocyte GR activity (Spearman $\rho=0.549, P<0.001$ ) and the erythrocyte GSH/GSSG ratio (Spearman $\rho=0.367, P=0.009$; Fig. 2B).

Erythrocyte GSH/GSSG ratio was negatively correlated with the plasma MDA level (Spearman $\rho=-0.370, P=0.008$; Supplemental Fig. 2A). The plasma selenium level was correlated with erythrocyte GPx activity (Spearman $\rho=0.353$, $P=0.012$; Supplemental Fig. 2B). The plasma T-GSH level was correlated with the erythrocyte GSH/GSSG ratio (Spearman $\rho=0.327, P=0.020$ ) and the GCLC level (Spearman $\rho=0.331$, $P=0.019$; Supplemental Fig. 2C). The erythrocyte GCLC level was not significantly correlated with the erythrocyte GSH/
A

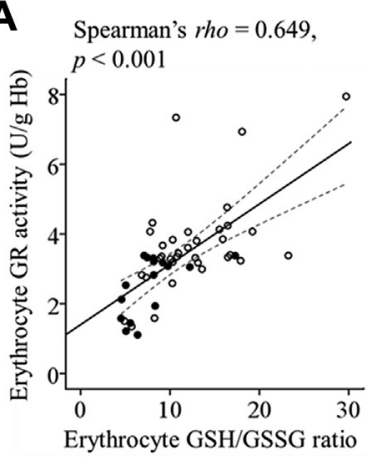

Survivors
B

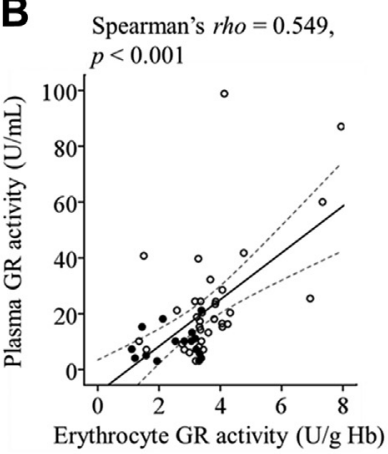

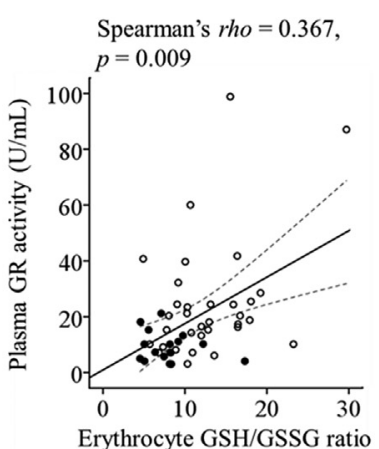

Erythrocyte GSH/GSSG ratio

Fig. 2 - Correlations between plasma GR activity, erythrocyte GR activity, and the erythrocyte GSH/GSSG ratio of septic shock patients. (A) Erythrocyte GR activity was correlated with the erythrocyte-reduced GSH/GSSG ratio. (B) Plasma GR activity was correlated with erythrocyte GR activity and the erythrocyte GSH/GSSG ratio. 
GSSG ratio (Spearman $\rho=0.277, \mathrm{P}=0.052$; Supplemental Fig. 2D).

\section{Discussion}

The present study showed that plasma GR activity was significantly correlated with erythrocyte GR activity and the erythrocyte GSH/GSSG ratio and that a decrease in plasma GR activity was independently associated with an increase in mortality both in endotoxemic rats and in patients with septic shock. The present study also showed that the erythrocyte GSH/GSSG ratio was negatively correlated with the plasma MDA level, which was significantly correlated with the erythrocyte $\mathrm{H}_{2} \mathrm{O}_{2}$ level. Previous studies have reported that the antioxidant defense mediated by GR, the reduction of GSSG to $\mathrm{GSH}$, is required for an effective innate immune response against bacterial infection [32]. These data indicate that plasma GR activity can be considered to be a useful parameter in the estimation of antioxidant capacities, particularly the erythrocyte GSH/GSSG ratio, and that the enhancement of GR activity may increase the GSH redox ratio, reduce oxidative stresses, and improve clinical outcomes in patients with septic shock.

In the present study, the plasma T-GSH level was significantly correlated with the erythrocyte GSH/GSSG ratio. However, the correlation of the plasma T-GSH level with the erythrocyte GSH/GSSG ratio was not as positive as that of plasma GR activity. Furthermore, our experimental data also showed that the erythrocyte GSH/GSSG ratio and erythrocyte and plasma GR activities in the HD-LPS group was significantly lower than those in the LD-LPS group, but the plasma T-GSH level in the HD-LPS group was not different from that in the LD-LPS group. To estimate the erythrocyte GSH/GSSG ratio, some researchers have tried to measure the plasma or whole blood T-GSH level and have reported that a decrease in the plasma or whole blood T-GSH level was associated with a decrease in the erythrocyte GSH/GSSG ratio and an increase in oxidative stresses [11,16,33]. Previous experimental studies reported that GCL activity was decreased during endotoxemia and a decrease in GCL activity might be primarily responsible for a decrease in the T-GSH level $[12,34,35]$. Our data showed that the plasma T-GSH level was also correlated with the erythrocyte GCLC level which was not correlated with the erythrocyte GSH/GSSG ratio. These data indicate that changes in the plasma T-GSH level are affected by the GSH synthesis ability and the GSH redox state. Therefore, we suggest that plasma GR activity is a more reliable parameter to estimate the erythrocyte GSH/GSSG ratio than the plasma T-GSH level.

In the present study, a decrease in the plasma selenium level was also associated with an increase in the 28-d mortality of patients with septic shock, and the plasma selenium level was significantly correlated with erythrocyte and plasma GPx activities. Selenium is an essential trace element to synthesize GPx, and its level is markedly depleted in critically ill patients [36,37]. To restore selenium depletion during septic shock, selenium treatment has been widely investigated. Some studies have shown that selenium improves the clinical outcomes of patients [38-42], but other studies failed to show survival benefits [43-45]. Our previous experimental study showed that selenium treatment significantly increases GPx activity in paraquatintoxicated rats, but it decreased the GSH/GSSG ratio and failed to improve survival [20]. We suggest that the limited benefits of selenium in patients with septic shock may be due to relative GSH depletion during GPx-induced oxidation of GSH to GSSG and that a selenium-only treatment may not be sufficient to overwhelm oxidative stresses during septic shock. The GSH redox ratio is maintained by the combination of ROS elimination by selenium (GPx) and GSH restoration by GR. The present study showed that a decrease in GR activity and a decrease in the selenium level were independently associated with an increase in mortality. If an adjunctive treatment to enhance GR activity is combined with selenium treatment, it may be more effective to reduce oxidative stresses and to improve clinical outcomes in patients with septic shock.

However, our clinical data were obtained from a small number of patients at a single center, and our experimental data were obtained from male endotoxemic rats. To confirm our data, large-scaled clinical studies and additional experimental studies using a clinically relevant sepsis model such as cecal ligation and puncture with antibiotics administration are needed [46].

\section{Conclusions}

We found that plasma GR activity was well correlated with erythrocyte GR activity and the erythrocyte GSH/GSSG ratio. Furthermore, we also found that a decrease in plasma GR activity was independently associated with an increase in the mortality of patients with septic shock.

\section{Acknowledgment}

The authors thank A Reum Lee for technical assistance.

This work was supported by a grant of the Korean Health Technology R\&D Project, Ministry of Health and Welfare, Republic of Korea (grant number: HI11C1420).

Authors' contributions: J.S.K., W.Y.K., G.J.S., and K.S.K. participated in the study design. W.Y.K., K.S.K., Y.S.J., and S.H.K. performed the experimental study. J.S.K., W.Y.K., G.J.S., and S.E.L. performed the clinical study. Y.S.J., S.H.K., and S.E.L. processed the data and performed the statistical analysis. J.S.K. and W.Y.K. wrote the article. J.S.K., W.Y.K., K.S.K., and G.J.S. critically revised the article for important intellectual content. All authors read and approved the final article.

\section{Disclosure}

There are no conflicts of interest. 


\section{Supplementary data}

Supplementary data related to this article can be found at http://dx.doi.org/10.1016/j.jss.2015.07.044

\section{R E F E R E N C E S}

[1] Cohen J. The immunopathogenesis of sepsis. Nature 2002;19: 885.

[2] Victor VM, Rocha M, De la Fuente M. Immune cells: free radicals and antioxidants in sepsis. Int Immunopharmacol 2004;4:327.

[3] Macdonald J, Galley HF, Webster NR. Oxidative stress and gene expression in sepsis. Br J Anaesth 2003;90:221.

[4] Huet O, Obata R, Aubron C, et al. Plasma-induced endothelial oxidative stress is related to the severity of septic shock. Crit Care Med 2007;35:821.

[5] Gloire G, Piette J. Redox regulation of nuclear posttranslational modifications during NF-kappaB activation. Antioxid Redox Signal 2009;11:2209.

[6] Bhoj VG, Chen ZJ. Ubiquitylation in innate and adaptive immunity. Nature 2009;458:430.

[7] Heffner JE, Repine JE. Pulmonary strategies of antioxidant defense. Am Rev Respir Dis 1989;140:531.

[8] Bridges AB, Scott NA, Pringle TH, McNeill GP, Belch JJ. Relationship between the extent of coronary artery disease and indicators of free radical activity. Clin Cardiol 1992; 15:169.

[9] Carbonell LF, Nadal JA, Llanos MC, Hernández I, Nava E, Díaz J. Depletion of liver glutathione potentiates the oxidative stress and decreases nitric oxide synthesis in a rat endotoxin shock model. Crit Care Med 2000; 28:2002.

[10] Lyons J, Rauh-Pfeiffer A, Ming-Yu Y, et al. Cysteine metabolism and whole blood glutathione synthesis in septic pediatric patients. Crit Care Med 2001;29:870.

[11] Németh I, Boda D. Xanthine oxidase activity and blood glutathione redox ratio in infants and children with septic shock syndrome. Intensive Care Med 2001;27:216.

[12] Lu SC. Regulation of hepatic glutathione synthesis: current concepts and controversies. FASEB J 1999;13:1169.

[13] Griffith OW, Meister A. Glutathione: interorgan translocation, turnover, and metabolism. Proc Natl Acad Sci U S A 1979;76:5606.

[14] Look MP, Rockstroh JK, Rao GS, et al. Serum selenium, plasma glutathione (GSH) and erythrocyte glutathione peroxidase (GSH-Px)-levels in asymptomatic versus symptomatic human immunodeficiency virus-1 (HIV-1)infection. Eur J Clin Nutr 1997;51:266.

[15] Harlan JM, Levine JD, Callahan KS, Schwartz BR, Harker LA. Glutathione redox cycle protects cultured endothelial cells against lysis by extracellularly generated hydrogen peroxide. J Clin Invest 1984;73:706.

[16] Unt E, Kairane C, Vaher I, Zilmer M. Red blood cell and whole blood glutathione redox status in endurance-trained men following a ski marathon. J Sports Sci Med 2008; 7:344.

[17] Curello S, Ceconi C, Cargnoni A, Cornacchiari A, Ferrari R, Albertini A. Improved procedure for determining glutathione in plasma as an index of myocardial oxidative stress. Clin Chem 1987;33:1448.

[18] Burk RF, Hill KE, Motley AK. Selenoprotein metabolism and function: evidence for more than one function for selenoprotein P. J Nutr 2003;133:1517S.
[19] Cheng W, Fu YX, Porres JM, Ross DA, Lei XG. Seleniumdependent cellular glutathione peroxidase protects mice against a pro-oxidant-induced oxidation of NADPH, NADH, lipids, and protein. FASEB J 1999;13:1467.

[20] Kim KS, Suh GJ, Kwon WY, et al. Antioxidant effects of selenium on lung injury in paraquat intoxicated rats. Clin Toxicol 2012;50:749.

[21] Comhair SA, Bhathena PR, Farver C, Thunnissen FB, Erzurum SC. Extracellular glutathione peroxidase induction in asthmatic lungs: evidence for redox regulation of expression in human airway epithelial cells. FASEB J 2001; 15:70.

[22] Wichmann MW, Inthorn D, Andress HJ, Schildberg FW. Incidence and mortality of severe sepsis in surgical intensive care patients: the influence of patient gender on disease process and outcome. Intensive Care Med 2000;26:167.

[23] Northern AL, Rutter SM, Peterson CM. Cyclic changes in the concentrations of peripheral blood immune cells during the normal menstrual cycle. Proc Soc Exp Biol Med 1994;207:81.

[24] Levy MM, Fink MP, Marshall JC, et al. SCCM/ESICM/ACCP/ ATS/SIS. 2001 SCCM/ESICM/ACCP/ATS/SIS International Sepsis Definitions Conference. Crit Care Med 2003;31: 1250.

[25] Dellinger RP, Levy MM, Carlet JM, et al. Surviving Sepsis Campaign: international guidelines for management of severe sepsis and septic shock: 2008. Crit Care Med 2008; 36:296.

[26] Knaus WA, Draper EA, Wagner DP, Zimmerman JE. APACHE II: a severity of disease classification system. Crit Care Med $1985 ; 13: 818$.

[27] Vincent JL, Moreno R, Takala J, et al. The SOFA (sepsis-related organ failure assessment) score to describe organ dysfunction/failure. On behalf of the working group on sepsis-related problems of the European Society of Intensive Care Medicine. Intensive Care Med 1996;22:707.

[28] Jain SK, Micinski D. Vitamin D upregulates glutamate cysteine ligase and glutathione reductase, and GSH formation, and decreases ROS and MCP-1 and IL-8 secretion in high-glucose exposed U937 monocytes. Biochem Biophys Res Commun 2013;437:7.

[29] Tamer L, Sucu N, Polat G, et al. Decreased serum total antioxidant status and erythrocyte-reduced glutathione levels are associated with increased serum malondialdehyde in atherosclerotic patients. Arch Med Res 2002;33:257.

[30] Tiran B, Tiran A, Rossipal E, Lorenz O. Simple decomposition procedure for determination of selenium in whole blood, serum and urine by hydride generation atomic absorption spectroscopy. J Trace Elem Electrolytes Health Dis 1993;7: 211.

[31] Durrleman S, Simon R. Flexible regression models with cubic splines. Stat Med 1989;8:551.

[32] Yan J, Ralston MM, Meng X, et al. Glutathione reductase is essential for host defense against bacterial infection. Free Radic Biol Med 2013;61:320.

[33] Morrison JA, Jacobsen DW, Sprecher DL, Robinson K, Khoury P, Daniels SR. Serum glutathione in adolescent males predicts parental coronary heart disease. Circulation 1999; 100:2244.

[34] Payabvash S, Ghahremani MH, Goliaei A, et al. Nitric oxide modulates glutathione synthesis during endotoxemia. Free Radic Biol Med 2006;41:1817.

[35] Tomasi ML, Ryoo M, Yang H, Iglesias Ara A, Ko KS, Lu SC. Molecular mechanisms of lipopolysaccharide-mediated inhibition of glutathione synthesis in mice. Free Radic Biol Med 2014;68:148.

[36] Sakr Y, Reinhart K, Bloos F, et al. Time course and relationship between plasma selenium concentrations, 
systemic inflammatory response, sepsis, and multiorgan failure. Br J Anaesth 2007;98:775.

[37] Forceville X, Vitoux D, Gauzit R, Combes A, Lahilaire P, Chappuis P. Selenium, systemic immune response syndrome, sepsis, and outcome in critically ill patients. Crit Care Med 1998;26:1536.

[38] Angstwurm MW, Engelmann L, Zimmermann T, et al. Selenium in intensive care (SIC): results of a prospective randomized, placebo-controlled, multiple-center study in patients with severe systemic inflammatory response syndrome, sepsis, and septic shock. Crit Care Med 2007;35:118.

[39] Alhazzani W, Jacobi J, Sindi A, et al. The effect of selenium therapy on mortality in patients with sepsis syndrome: a systematic review and meta-analysis of randomized controlled trials. Crit Care Med 2013;41:1555.

[40] Manzanares W, Dhaliwal R, Jiang X, Murch L, Heyland DK. Antioxidant micronutrients in the critically ill: a systematic review and meta-analysis. Crit Care 2012;16:R66.

[41] Huang TS, Shyu YC, Chen HY, et al. Effect of parenteral selenium supplementation in critically ill patients: a systematic review and meta-analysis. PLoS One 2013;8:e54431.
[42] Landucci F, Mancinelli P, De Gaudio AR, Virgili G. Selenium supplementation in critically ill patients: a systematic review and meta-analysis. J Crit Care 2014;29:150.

[43] Andrews PJ, Avenell A, Noble DW, et al. Scottish Intensive care Glutamine or seleNium Evaluative Trial Trials Group. Randomised trial of glutamine, selenium, or both, to supplement parenteral nutrition for critically ill patients. BMJ 2011;342:d1542.

[44] Heyland D, Muscedere J, Wischmeyer PE, et al. Canadian Critical Care Trials Group. A randomized trial of glutamine and antioxidants in critically ill patients. N Engl J Med 2013; 368:1489.

[45] Forceville X, Laviolle B, Annane D, et al. Effects of high doses of selenium, as sodium selenite, in septic shock: a placebocontrolled, randomized, double-blind, phase II study. Crit Care 2007;11:R73.

[46] Kotake Y, Moore DR, Vasquez-Walden A, Tabatabaie T, Sang H. Antioxidant amplifies antibiotic protection in the cecal ligation and puncture model of microbial sepsis through interleukin-10 production. Shock 2003; 19:252. 